\title{
CHAOS IN PERTURBED LOTKA-VOLTERRA SYSTEMS
}

\author{
J. R. CHRISTIE ${ }^{1}, \mathrm{~K}$. GOPALSAMY ${ }^{1}$ and JIBIN LI
}

(Received 24 March 1997; revised 17 November 1999)

\begin{abstract}
Lotka-Volterra systems have been used extensively in modelling population dynamics. In this paper, it is shown that chaotic behaviour in the sense of Smale can exist in timeperiodically perturbed systems of Lotka-Volterra equations. First, a slowly varying threedimensional perturbed Lotka-Volterra system is considered and the corresponding unperturbed system is shown to possess a heteroclinic cycle. By using Melnikov's method, sufficient conditions are obtained for the perturbed system to have a transverse heteroclinic cycle and hence to possess chaotic behaviour in the sense of Smale. Then a special case involving a reduction to a two-dimensional Lotka-Volterra system is examined, leading finally to an application with a model for the self-organisation of macromolecules.
\end{abstract}

\section{Introduction}

Lotka-Volterra systems and many of their variants have been the subject of numerous studies. Many authors have investigated the potential chaotic behaviour of certain systems of Lotka-Volterra equations (see for instance $[4,6,10,11,14-16,20,21]$ ) and a large number of authors have used computer simulations in their investigations. There are not many analytical methods available to establish the existence of chaotic behaviour in systems of differential equations. However, if we have an autonomous system of ordinary differential equations possessing a heteroclinic cycle or a homoclinic orbit, then we can study such systems under a time-periodic perturbation by using a technique originally due to Melnikov [13]. This technique is called Melnikov's method and it can be used to establish the existence of a transverse heteroclinic cycle or a transverse homoclinic point in the dynamics generated by the associated Poincaré map [7,23]; the existence of such a cycle or point then implies the existence of chaotic

\footnotetext{
'Department of Mathematics and Statistics, The Flinders University of South Australia, GPO Box 2100, Adelaide, South Australia, 5001, Australia; e-mails: johnc and gopal@ist.finders.edu.au.

${ }^{2}$ Institute of Applied Mathematics of Yunnan Province, Department of Mathematics, Kunming University of Technology, Yunnan 650093, People's Republic of China; e-mail: jibinli@ynu.edu.cn. (c) Australian Mathematical Society 2001, Serial-fee code 0334-2700/01
} 
behaviour in the sense of Smale by the Smale-Birkhoff homoclinic theorem $[24,27]$. The authors have given an application of Melnikov's method to a sociobiological system in a recent paper [2].

The study of Lotka-Volterra systems driven by time-periodic external forces is of considerable interest in applications since almost all communities (macroscopic and microscopic) of natural populations are in periodic environments (seasons, daylight, temperature, mating habits, food availability). One of the primary concerns is to examine and understand how the magnitude of perturbations affect the densities of various species in different communities. A wide variety of dynamical behaviour of systems in periodic environments is possible [5]. The primary purpose of this article is to use Melnikov's method to investigate the potential existence of chaotic behaviour in certain Lotka-Volterra systems driven by time-periodic perturbations.

The layout of the paper is as follows. In Section 2, we consider a slowly varying perturbed three-dimensional Lotka-Volterra system and show that the corresponding unperturbed system has a heteroclinic cycle and a continuous family of periodic orbits. In Section 3, we use the results of Wiggins and Holmes [25] to apply Melnikov's method. Sufficient conditions for the existence of chaotic behaviour in the sense of Smale are obtained. Then, in Section 4, we consider a special case of the threedimensional Lotka-Volterra system which we reduce to a two-dimensional system. Finally, in Section 5, an application is given with a model for the self-organisation of macromolecules. A sufficient condition for the existence of chaos is provided.

\section{The Lotka-Volterra system}

Consider the slowly varying time-periodically perturbed Lotka-Volterra system

$$
\begin{aligned}
& \frac{d x}{d t}=x(c-b x-2 d y+a z)+\epsilon\left[\lambda_{1} x+\left(\lambda_{2}+\lambda_{3} x\right) \sin \omega t\right], \\
& \frac{d y}{d t}=y(-c+2 b x+d y-a z)+\epsilon\left[\lambda_{1} y+\left(\lambda_{2}+\lambda_{3} y\right) \sin \omega t\right], \\
& \frac{d z}{d t}=\epsilon z\left(\lambda_{4}+\lambda_{5} \sin \omega t-\lambda_{6} x+\lambda_{7} y-\lambda_{8} z\right),
\end{aligned}
$$

where $0 \leq \epsilon \ll 1$ denotes the perturbation parameter, $\omega>0$ is the frequency of the perturbation, $a, b, c, d>0$ and $\lambda_{j}(j=1, \ldots, 8)$ are real parameters. Letting $\phi(t)=\omega t(\bmod T)$, where $T=2 \pi / \omega$ is the period of the perturbation, we have the suspended system

$$
\begin{aligned}
& \frac{d x}{d t}=x(c-b x-2 d y+a z)+\epsilon\left[\lambda_{1} x+\left(\lambda_{2}+\lambda_{3} x\right) \sin \phi\right], \\
& \frac{d y}{d t}=y(-c+2 b x+d y-a z)+\epsilon\left[\lambda_{1} y+\left(\lambda_{2}+\lambda_{3} y\right) \sin \phi\right],
\end{aligned}
$$




$$
\begin{aligned}
\frac{d z}{d t} & =\epsilon z\left(\lambda_{4}+\lambda_{5} \sin \phi-\lambda_{6} x+\lambda_{7} y-\lambda_{8} z\right), \\
\frac{d \phi}{d t} & =\omega,
\end{aligned}
$$

where $(x, y, z, \phi) \in \mathbb{R}^{3} \times S_{2 \pi}$, in which $S_{2 \pi}=\mathbb{R}(\bmod 2 \pi)$. Now $(2.1)$ with $\epsilon=0$ is a one-parameter family of planar Hamiltonian systems with Hamiltonian

$$
H(x, y, z)=x y(c-b x-d y+a z)=h .
$$

In the plane $z=z_{0}$, there are four fixed points: $\mathrm{A}(0,0), \mathrm{B}(0, p / d)$ and $\mathrm{C}(p / b, 0)$ are saddle points; $\mathrm{D}(p /(3 b), p /(3 d))$ is a centre, where $p=c+a z_{0}$. Consider $x \geq 0$, $y \geq 0$ and $z \geq 0$. The straight lines $x=0, y=0$ and $p-d y-b x=0$ are invariant lines. When $h \in\left(0, p^{3} /(27 b d)\right)$, the family of curves defined by (2.3) is a continuous periodic family $\left\{\Gamma^{h, z}\right\}$ surrounding the centre at $D$. The system (2.1) for $\epsilon=0$ has the phase portrait shown in Figure 1(a). There are three heteroclinic orbits consisting of three straight line segments connecting the three saddle points. The existence of this heteroclinic cycle enables us to consider an application of Melnikov's method in the next section. One can derive the following parametric representations for the three heteroclinic orbits (each orbit lies on the plane $z=z_{0}$ ):

$$
\begin{aligned}
& \mathrm{AB}: \quad x_{0}(t)=0, \quad y_{0}(t)=\frac{c+a z}{d(1+\exp ((c+a z) t))} \\
& \mathrm{AC}: \quad x_{0}(t)=\frac{c+a z}{b(1+\exp (-(c+a z) t))}, \quad y_{0}(t)=0, \\
& \mathrm{BC}: \quad x_{0}(t)=\frac{c+a z}{b(1+\exp ((c+a z) t))}, \quad y_{0}(t)=\frac{c+a z}{d(1+\exp (-(c+a z) t))},
\end{aligned}
$$

and the periodic family $\left\{\Gamma^{h, z}\right\}$ has parameterisation:

$$
\begin{aligned}
& x_{k}(t)=\frac{\gamma}{1-A^{2} \operatorname{sn}^{2}(\Omega t, k)}, \\
& y_{k}(t)=\frac{1}{2 d}\left[c-\frac{b \gamma}{1-A^{2} \operatorname{sn}^{2}(\Omega t, k)}+a z-\frac{2 A^{2} \Omega \operatorname{sn}(\Omega t, k) \operatorname{cn}(\Omega t, k) \operatorname{dn}(\Omega t, k)}{1-A^{2} \operatorname{sn}^{2}(\Omega t, k)}\right], \\
& z_{k}(t)=z_{0},
\end{aligned}
$$

where $A=(\beta-\gamma) / \beta, \Omega=\sqrt{b(\alpha-\gamma) \beta / 2}$ and $\alpha, \beta$ and $\gamma$ are the real zeros of the polynomial

$$
G(u)=u^{3}-\frac{2}{b}(c+a z) u^{2}+\frac{1}{b^{2}}(c+a z)^{2} u-\frac{4 d h}{b^{2}},
$$

for fixed $z=z_{0}$, and $\alpha>p / b>\beta>p /(3 b)>\gamma>0$. The functions $\operatorname{sn}(\cdot, k), \operatorname{cn}(\cdot, k)$ and $\operatorname{dn}(\cdot, k)$ are Jacobi elliptic functions [1] with elliptic modulus $k=\sqrt{(\beta-\gamma) \alpha /[(\alpha-\gamma) \beta]}, 0<A<k$. The period of the family $\left\{\Gamma^{h, z}\right\}$ is 
$T^{h, z}=2 K(k) / \Omega$, where $k \in(0,1)$, in which $K(k)$ is the complete elliptic integral of the first kind [1]. As $h$ varies from 0 to $p^{3} /(27 b d), k$ decreases from 1 to 0 and $\left\{\Gamma^{h, z}\right\}$ contracts to the centre $\mathrm{D}$. The period of $\left\{\Gamma^{h, z}\right\}$ is a monotonic function of $k$. As $k$ increases from 0 to 1 , the period of $\left\{\Gamma^{h, z}\right\}$ increases from 0 to $\infty$.

\section{Melnikov integrals}

Wiggins and Holmes [25,26] have studied periodic orbits and homoclinic orbits in slowly varying oscillators (see also $[3,18,19,22,27]$ ). Generally, for heteroclinic orbits, the Melnikov function for homoclinic orbits cannot work because $(\partial H / \partial z)\left(q_{0}(-\infty)\right) \neq(\partial H / \partial z)\left(q_{0}(\infty)\right)$, where $q_{0}(t)=\left(x_{0}(t), y_{0}(t), z_{0}\right)$ (see [25]). However, for the system (2.2) with $\epsilon=0$, since $\partial H / \partial z=a x y$, by (2.4)-(2.6) we know that $(\partial H / \partial z)\left(q_{0}(-\infty)\right)=(\partial H / \partial z)\left(q_{0}(\infty)\right)=0$, so that we can use the same formula for the Melnikov function for homoclinic orbits with heteroclinic orbits:

$$
\begin{aligned}
M\left(t_{0}\right)= & \int_{-\infty}^{\infty}\left(\frac{\partial H}{\partial x}\left(q_{0}(t)\right) g_{1}\left(q_{0}(t), t+t_{0}\right)+\frac{\partial H}{\partial y}\left(q_{0}(t)\right) g_{2}\left(q_{0}(t), t+t_{0}\right)\right. \\
& \left.+\frac{\partial H}{\partial z}\left(q_{0}(t)\right) g_{3}\left(q_{0}(t), t+t_{0}\right)\right) d t-\frac{\partial H}{\partial z}\left(\gamma\left(z_{0}\right)\right) \int_{-\infty}^{\infty} g_{3}\left(q_{0}(t), t+t_{0}\right) d t,
\end{aligned}
$$

where $\gamma$ is the parameterisation of a union of saddle points of the one-parameter family of planar systems and $g=\left(g_{1}, g_{2}, g_{3}\right)$ is the perturbation function (see [25]). Since the points $A, B$ and $C$ are saddle points of (2.1) with $\epsilon=0$, when viewed in the three-dimensional phase space, this system possesses three normally hyperbolic invariant one-dimensional manifolds $\mathscr{N}_{i}(i=1,2,3)$, given by the union of saddle points of the one-parameter family of planar systems. For each $i=1,2,3, \mathscr{N}_{i}$ has twodimensional global stable and unstable manifolds $W^{s}\left(\mathscr{N}_{i}\right)$ and $W^{u}\left(\mathscr{N}_{i}\right)$ respectively such that $\bigcup_{i=1}^{3} W^{s}\left(\mathscr{N}_{i}\right) \cap W^{u}\left(\mathscr{N}_{i}\right)$ is made up of the union of the heteroclinic orbits of the one-parameter family of planar systems (2.1) with $\epsilon=0$ which is shown in Figure 1(b).

For the suspended system (2.2) with $\epsilon=0$, we denote the normally hyperbolic invariant sets by $\mathscr{M}_{i} \equiv\left(\mathscr{N}_{i}, \phi\right)=\mathscr{N}_{i} \times S_{2 \pi}$. By the implicit function theorem,

$$
\begin{aligned}
\mathscr{M}_{i}= & \left\{\left(\gamma_{i}(z), \phi\right): \gamma_{i}(z)=(x(z), y(z), z), f_{1}(x, y, z)=f_{2}(x, y, z)=0,\right. \\
& \left.\left.\frac{\partial\left(f_{1}, f_{2}\right)}{\partial(x, y)}\right|_{y_{i}(z)}<0, \phi \in S_{2 \pi}, z \in(0, Z)\right\},
\end{aligned}
$$

for $i=1,2,3$, where $\left(f_{1}, f_{2}, 0\right)$ is the unperturbed vector field, and

$$
\gamma_{1}(z)=\left(\frac{c+a z}{b}, 0, z\right), \quad \gamma_{2}(z)=(0,0, z), \quad \gamma_{3}(z)=\left(0, \frac{c+a z}{d}, z\right)
$$




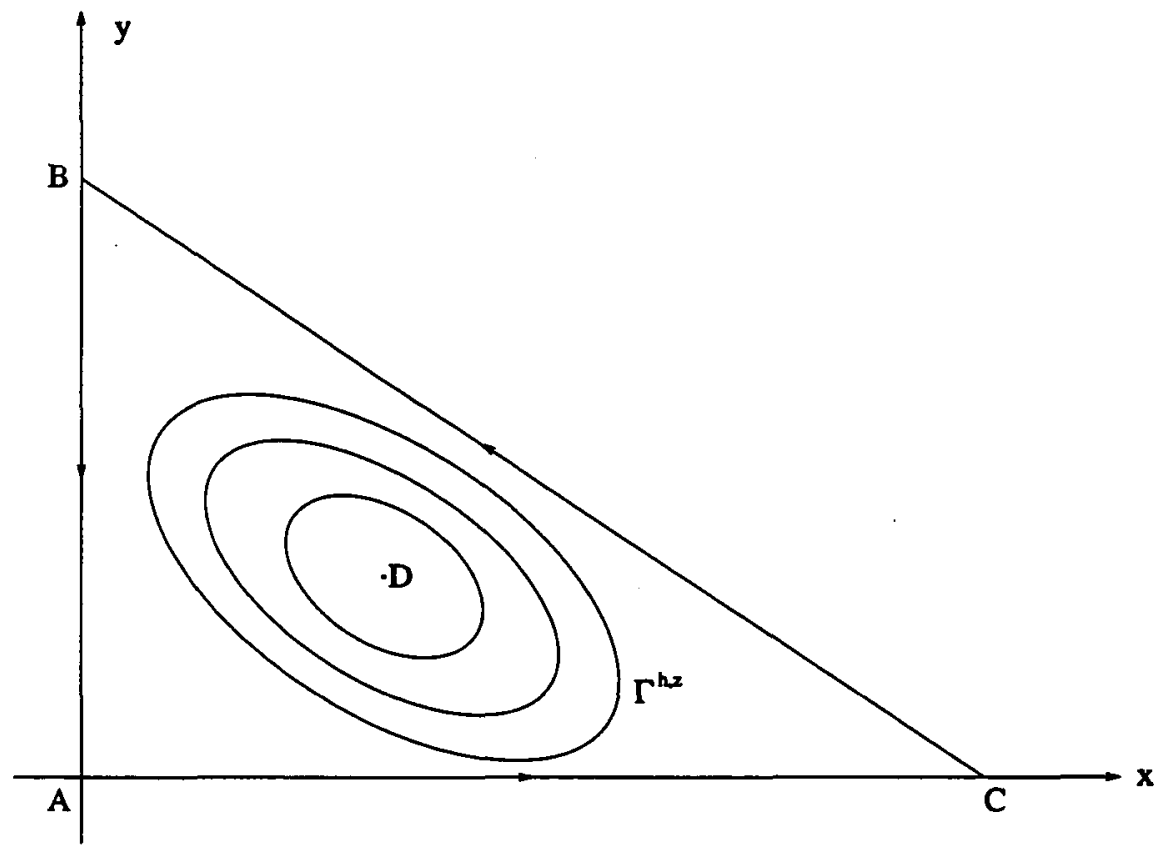

(a)

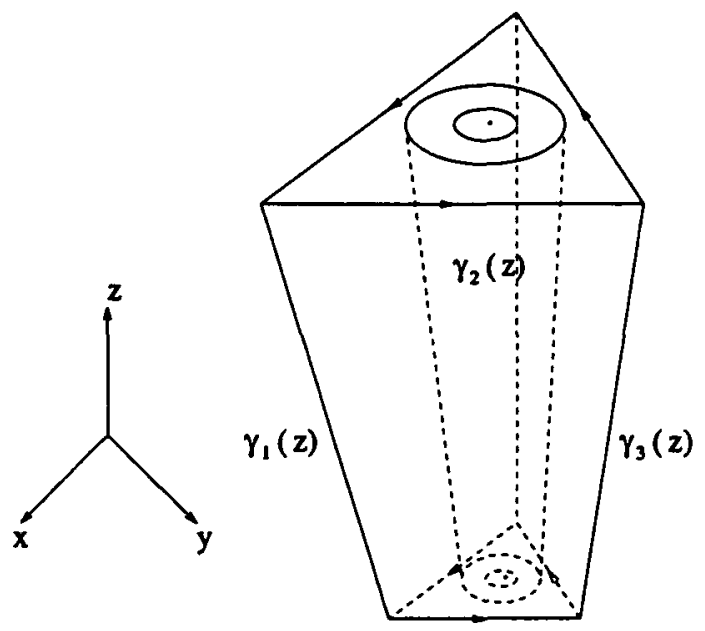

(b)

FIGURE 1. Phase portrait of the system (2.1) with $\epsilon=0$. 
and $Z>0$ is a constant. By Proposition 2.1 of Wiggins and Holmes [25], we know that there exists $\epsilon_{0}>0$ such that for $0<\epsilon<\epsilon_{0} \ll 1$ there exist three normally hyperbolic invariant one-manifolds

$$
\mathscr{M}_{\epsilon_{i}}=\left\{\left(\gamma_{i}(z, \phi ; \epsilon), \phi\right)=\left(\gamma_{i}(z)+O(\epsilon), \phi\right): \phi \in S_{2 \pi}, z \in(0, Z)\right\}, \quad i=1,2,3 .
$$

Each $\mathscr{M}_{\epsilon_{i}}$ has local stable and unstable manifolds denoted by $W_{10 c}^{s}\left(\mathscr{M}_{\epsilon_{i}}\right)$ and $W_{10}^{u}\left(\mathscr{M}_{\epsilon_{i}}\right)$ respectively, which are $C^{r}$-close to the local stable and unstable manifolds of $\mathscr{M}_{i}$, denoted by $W_{10 c}^{s}\left(\mathscr{M}_{i}\right)$ and $W_{\text {loc }}^{u}\left(\mathscr{M}_{i}\right)$, respectively. We now use Proposition 2.2 of Wiggins and Holmes [25], a result which involves averaging. Let $\overline{g_{3}\left(\gamma_{i}(z)\right)}=0$, $i=1,2,3$, where $\overline{g_{3}}$ is the time average of $g_{3}$. Then, using the fact that

$$
g_{3}(x, y, z, t)=z\left(\lambda_{4}+\lambda_{5} \sin \omega t-\lambda_{6} x+\lambda_{7} y-\lambda_{8} z\right),
$$

we have

$$
\begin{aligned}
& \overline{g_{3}\left(\gamma_{1}(z)\right)}=z\left(\lambda_{4}-\lambda_{6} \frac{c+a z}{b}-\lambda_{8} z\right)=0, \\
& \overline{g_{3}\left(\gamma_{2}(z)\right)}=z\left(\lambda_{4}-\lambda_{8} z\right)=0 \\
& \overline{g_{3}\left(\gamma_{3}(z)\right)}=z\left(\lambda_{4}+\lambda_{7} \frac{c+a z}{d}-\lambda_{8} z\right)=0 .
\end{aligned}
$$

Hence we obtain:

(1) When $\lambda_{4} \neq c \lambda_{6} / b$, at the points $z_{0}=0$ and $z_{0}=z_{1}=\left(b \lambda_{4}-c \lambda_{6}\right) /\left(a \lambda_{6}+b \lambda_{8}\right)$ (if $\left.a \lambda_{6}+b \lambda_{8} \neq 0\right), \overline{g_{3}\left(\gamma_{1}\left(z_{0}\right)\right)}=0$ and $d \overline{\left(g_{3}\left(\gamma_{1}(z)\right)\right)} /\left.d z\right|_{z=z_{0}} \neq 0$, so $\left(\gamma_{1}\left(z_{0}\right)+O(\epsilon), \phi\right)$ is a hyperbolic periodic orbit on $\mathscr{M}_{\epsilon_{1}}$ with period $2 \pi / \omega$.

(2) When $\lambda_{4} \neq 0$, at the points $z_{0}=0$ and $z_{0}=z_{2}=\lambda_{4} / \lambda_{8}$ (if $\left.\lambda_{8} \neq 0\right), \overline{g_{3}\left(\gamma_{2}\left(z_{0}\right)\right)}=$ 0 and $d\left(\overline{g_{3}\left(\gamma_{2}(z)\right)}\right) /\left.d z\right|_{z=z_{0}} \neq 0$, so $\left(\gamma_{2}\left(z_{0}\right)+O(\epsilon), \phi\right)$ is a hyperbolic periodic orbit on $\mathscr{M}_{\epsilon_{2}}$ with period $2 \pi / \omega$.

(3) When $\lambda_{4} \neq-c \lambda_{7} / d$, at the points $z_{0}=0$ and $z_{0}=z_{3}=\left(d \lambda_{4}+c \lambda_{7}\right) /\left(d \lambda_{8}-a \lambda_{7}\right)$ (if $d \lambda_{8}-a \lambda_{7} \neq 0$ ), $\overline{g_{3}\left(\gamma_{3}\left(z_{0}\right)\right)}=0$ and $d\left(\overline{g_{3}\left(\gamma_{3}(z)\right)}\right) /\left.d z\right|_{z=z_{0}} \neq 0$, so $\left(\gamma_{3}\left(z_{0}\right)+O(\epsilon), \phi\right)$ is a hyperbolic periodic orbit on $\mathscr{M}_{\epsilon_{3}}$ with period $2 \pi / \omega$.

In order to have the existence of a transverse heteroclinic cycle, we have to assume that three unperturbed heteroclinic orbits lie on the same $z_{0}$ level, so that we need the condition $z_{0}=z_{1}=z_{2}=z_{3}$ or $z_{0}=0$. From the first condition, it follows that $\lambda_{6}=0$ and $\lambda_{7}=0$. Hence, $z_{1}=z_{2}=z_{3}=\lambda_{4} / \lambda_{8}=z^{*}$, say, if $\lambda_{8} \neq 0$.

We now calculate the Melnikov functions. Along with the unperturbed heteroclinic orbits given by (2.4)-(2.6), from (3.1) the Melnikov functions are as follows (note that $\left.(\partial H / \partial z)\left(\gamma_{i}\left(z_{0}\right)\right)=0, i=1,2,3\right)$ :

$$
M_{A B}\left(t_{0}\right)=\int_{-\infty}^{\infty}\left\{x_{0}(t)\left(p-b x_{0}(t)-2 d y_{0}(t)\right)\left[\lambda_{1} y_{0}(t)+\left(\lambda_{2}+\lambda_{3} y_{0}(t)\right) \sin \left(\omega\left(t+t_{0}\right)\right)\right]\right.
$$




$$
\begin{aligned}
& +y_{0}(t)\left(p-2 b x_{0}(t)-d y_{0}(t)\right)\left[\lambda_{1} x_{0}(t)+\left(\lambda_{2}+\lambda_{3} x_{0}(t)\right) \sin \left(\omega\left(t+t_{0}\right)\right)\right] \\
& \left.+a x_{0}(t) y_{0}(t) z_{0}\left(\lambda_{4}+\lambda_{5} \sin \left(\omega\left(t+t_{0}\right)\right)-\lambda_{6} x_{0}(t)+\lambda_{7} y_{0}(t)-\lambda_{8} z_{0}\right)\right] d t \\
= & \lambda_{2} \int_{-\infty}^{\infty} y_{0}(t)\left(p-d y_{0}(t)\right) \sin \left(\omega\left(t+t_{0}\right)\right) d t \\
= & \frac{\lambda_{2} p^{2}}{d} \int_{-\infty}^{\infty} \frac{\sin \left(\omega\left(t+t_{0}\right)\right)}{1+e^{p t}}\left(1-\frac{1}{1+e^{p t}}\right) d t \\
= & \frac{\lambda_{2} p^{2}}{d}\left[\sin \omega t_{0} \int_{-\infty}^{\infty}\left(\frac{\cos \omega t}{1+e^{p t}}-\frac{\cos \omega t}{\left(1+e^{p t}\right)^{2}}\right) d t\right. \\
& \left.\quad+\cos \omega t_{0} \int_{-\infty}^{\infty}\left(\frac{\sin \omega t}{1+e^{p t}}-\frac{\sin \omega t}{\left(1+e^{p t}\right)^{2}}\right) d t\right] \\
= & \frac{\pi \omega \lambda_{2}}{d} \operatorname{cosech}\left(\frac{\pi \omega}{p}\right) \sin \omega t_{0},
\end{aligned}
$$

using a result from [1] and since the integrand of the second integral is odd. Similarly, we have

$$
\begin{aligned}
M_{A C}\left(t_{0}\right) & =\lambda_{2} \int_{-\infty}^{\infty} x_{0}(t)\left(p-b x_{0}(t)\right) \sin \left(\omega\left(t+t_{0}\right)\right) d t \\
& =\frac{\lambda_{2} p^{2}}{b} \int_{-\infty}^{\infty} \frac{\sin \left(\omega\left(t+t_{0}\right)\right)}{1+e^{-p t}}\left(1-\frac{1}{1+e^{-p t}}\right) d t \\
& =\frac{\pi \omega \lambda_{2}}{b} \operatorname{cosech}\left(\frac{\pi \omega}{p}\right) \sin \omega t_{0} .
\end{aligned}
$$

Also, we have

$$
\begin{aligned}
M_{B C}\left(t_{0}\right)= & \lambda_{2} \int_{-\infty}^{\infty}\left[x_{0}(t)\left(p-b x_{0}(t)-2 d y_{0}(t)\right)\right. \\
& \left.+y_{0}(t)\left(p-2 b x_{0}(t)-d y_{0}(t)\right)\right] \sin \left(\omega\left(t+t_{0}\right)\right) d t \\
& -\int_{-\infty}^{\infty} x_{0}(t) y_{0}(t) p\left(\lambda_{1}+\lambda_{3} \sin \left(\omega\left(t+t_{0}\right)\right)\right) d t \\
& +a z_{0} \int_{-\infty}^{\infty} x_{0}(t) y_{0}(t)\left(\lambda_{4}+\lambda_{5} \sin \left(\omega\left(t+t_{0}\right)\right)-\lambda_{6} x_{0}(t)+\lambda_{7} y_{0}(t)-\lambda_{8} z_{0}\right) d t \\
= & \frac{\lambda_{2} p^{2}}{b} \int_{-\infty}^{\infty} \frac{\sin \left(\omega\left(t+t_{0}\right)\right)}{1+e^{p t}}\left(1-\frac{1}{1+e^{p t}}-\frac{2}{1+e^{-p t}}\right) d t \\
& +\frac{\lambda_{2} p^{2}}{d} \int_{-\infty}^{\infty} \frac{\sin \left(\omega\left(t+t_{0}\right)\right)}{1+e^{-p t}}\left(1-\frac{2}{1+e^{p t}}-\frac{1}{1+e^{-p t}}\right) d t \\
& -\frac{p^{3}}{b d} \int_{-\infty}^{\infty} \frac{\lambda_{1}+\lambda_{3} \sin \left(\omega\left(t+t_{0}\right)\right)}{\left(1+e^{-p t}\right)\left(1+e^{p t}\right)} d t \\
& +\frac{a z_{0} p^{2}}{b d} \int_{-\infty}^{\infty} \frac{\lambda_{4}+\lambda_{5} \sin \left(\omega\left(t+t_{0}\right)\right)-\lambda_{8} z_{0}}{\left(1+e^{-p t}\right)\left(1+e^{p t}\right)} d t
\end{aligned}
$$




$$
\begin{aligned}
& -\frac{a z_{0} \lambda_{6} p^{3}}{b^{2} d} \int_{-\infty}^{\infty} \frac{d t}{\left(1+e^{p t}\right)^{2}\left(1+e^{-p t}\right)} \\
& +\frac{a z_{0} \lambda_{7} p^{3}}{b d^{2}} \int_{-\infty}^{\infty} \frac{d t}{\left(1+e^{p t}\right)\left(1+e^{-p t}\right)^{2}} \\
= & \frac{a z_{0} p\left(\lambda_{4}-\lambda_{8} z_{0}\right)}{b d}-\frac{\lambda_{1} p^{2}}{b d}+\frac{a z_{0} p^{2}}{2 b d}\left(\frac{\lambda_{7}}{d}-\frac{\lambda_{6}}{b}\right) \\
& -\left[\lambda_{2}\left(\frac{1}{b}+\frac{1}{d}\right)+\frac{\lambda_{3} p}{b d}-\frac{\lambda_{5} a z_{0}}{b d}\right] \pi \omega \operatorname{cosech}\left(\frac{\pi \omega}{p}\right) \sin \omega t_{0} .
\end{aligned}
$$

Therefore, when $z_{0}=0$,

$$
\begin{aligned}
& M_{A B}\left(t_{0}\right)=\frac{\pi \omega \lambda_{2}}{d} \operatorname{cosech}\left(\frac{\pi \omega}{c}\right) \sin \omega t_{0}, \\
& M_{A C}\left(t_{0}\right)=\frac{\pi \omega \lambda_{2}}{b} \operatorname{cosech}\left(\frac{\pi \omega}{c}\right) \sin \omega t_{0} \\
& M_{B C}\left(t_{0}\right)=-\frac{\lambda_{1} c^{2}}{b d}-\left[\lambda_{2}\left(\frac{1}{b}+\frac{1}{d}\right)+\frac{\lambda_{3} c}{b d}\right] \pi \omega \operatorname{cosech}\left(\frac{\pi \omega}{c}\right) \sin \omega t_{0}
\end{aligned}
$$

When $z_{0}=z^{*}$ (and hence $\lambda_{6}=\lambda_{7}=0$ and $\lambda_{8} \neq 0$ ),

$$
\begin{aligned}
& M_{A B}\left(t_{0}\right)=\frac{\pi \omega \lambda_{2}}{d} \operatorname{cosech}\left(A_{4} \omega\right) \sin \omega t_{0}, \\
& M_{A C}\left(t_{0}\right)=\frac{\pi \omega \lambda_{2}}{b} \operatorname{cosech}\left(A_{4} \omega\right) \sin \omega t_{0}, \\
& M_{B C}\left(t_{0}\right)=-A_{1} \lambda_{1}-\left(A_{2} \lambda_{2}+A_{3} \lambda_{3}+A_{5} \lambda_{5}\right) \pi \omega \operatorname{cosech}\left(A_{4} \omega\right) \sin \omega t_{0},
\end{aligned}
$$

where

$$
\begin{gathered}
A_{1}=\frac{\left(c \lambda_{8}+a \lambda_{4}\right)^{2}}{b d \lambda_{8}^{2}}, \quad A_{2}=\frac{1}{b}+\frac{1}{d}, \quad A_{3}=\frac{c \lambda_{8}+a \lambda_{4}}{b d \lambda_{8}} \\
A_{4}=\frac{\pi \lambda_{8}}{c \lambda_{8}+a \lambda_{4}}, \quad A_{5}=-\frac{a \lambda_{4}}{b d \lambda_{8}}
\end{gathered}
$$

From (3.2), we know that if $\lambda_{2} \neq 0$ and

$$
\left|\lambda_{1}\right|<\left|\frac{b+d}{c^{2}} \lambda_{2}+\frac{1}{c} \lambda_{3}\right| \pi \omega \operatorname{cosech}\left(\frac{\pi \omega}{c}\right),
$$

then every Melnikov function has simple zeros; hence, there exists a transverse heteroclinic cycle to the hyperbolic periodic orbits $\left(\gamma_{i}(0)+O(\epsilon), \phi\right), i=1,2,3$, for sufficiently small $\epsilon$. Similarly, from (3.3), if $\lambda_{2} \neq 0, \lambda_{8} \neq 0$ and

$$
A_{1}\left|\lambda_{1}\right|<\left|A_{2} \lambda_{2}+A_{3} \lambda_{3}+A_{5} \lambda_{5}\right| \pi \omega \operatorname{cosech}\left(A_{4} \omega\right)
$$




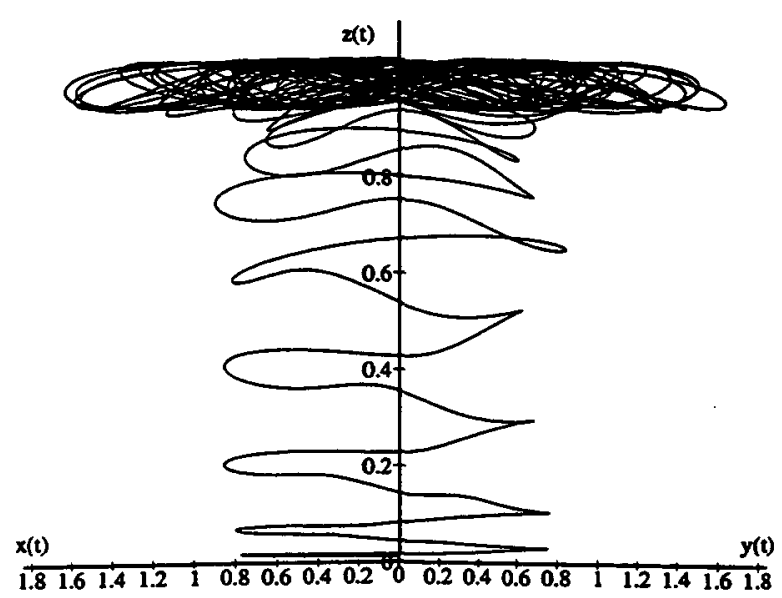

FIGURE 2. An orbit of (2.1) for $a=b=c=d=0.5, \omega=1, \lambda_{1}=-0.001, \lambda_{2}=1, \lambda_{3}=0, \lambda_{4}=1$, $\lambda_{5}=1, \lambda_{6}=0, \lambda_{7}=0, \lambda_{8}=1$ and $\epsilon=0.05$. The initial value chosen was $(x(0), y(0), z(0))=$ $(0,0,0.01)$.

then there exists a transverse heteroclinic cycle to the hyperbolic periodic orbits $\left(\gamma_{i}\left(z^{*}\right)+O(\epsilon), \phi\right), i=1,2,3$, for sufficiently small $\epsilon$. In both cases, the Poincare map of (2.2) has transverse homoclinic points; this means that (2.2) and hence (2.1) have chaotic motions of Smale horseshoe type for sufficiently small $\epsilon$ by the SmaleBirkhoff homoclinic theorem $[24,27]$.

THEOREM 3.1. Suppose $\lambda_{2} \neq 0$. If

(1) $\lambda_{4} \neq 0, c \lambda_{6} / b,-c \lambda_{7} / d$ and (3.4) holds, or

(2) $\lambda_{4} \neq 0, \lambda_{6}=\lambda_{7}=0, \lambda_{8} \neq 0$ and (3.5) holds,

then chaotic behaviour in the sense of Smale exists in the system (2.1) for sufficiently small $\epsilon$.

Consider the case when $a=b=c=d=0.5, \omega=1, \lambda_{1}=-0.001, \lambda_{2}=1$, $\lambda_{3}=0, \lambda_{4}=1, \lambda_{5}=1, \lambda_{6}=0, \lambda_{7}=0$ and $\lambda_{8}=1$. These parameter values imply that $A_{1}=4, A_{2}=4, A_{3}=4, A_{4}=\pi, A_{5}=-2$ and $z^{*}=1$ and it is easily verified that the hypotheses (1) and (2) in Theorem 3.1 are satisfied, so chaotic behaviour exists for sufficiently small $\epsilon$. We now present a computer simulation in Figure 2 where we have chosen $\epsilon=0.05$; this figure shows an orbit of (2.1) near the plane $z=1$. Clearly, the plane $z=0$ is invariant so orbits that start on $z=0$ never leave $z=0$ and, in effect, we have a two-dimensional system which is analysed in the next section. 


\section{The two-dimensional case}

Consider the system (2.1) with $z=0$. We obtain the two-dimensional timeperiodically perturbed Lotka-Volterra system given by

$$
\begin{aligned}
& \frac{d x}{d t}=x(c-b x-2 d y)+\epsilon\left[\lambda_{1} x+\left(\lambda_{2}+\lambda_{3} x\right) \sin \omega t\right], \\
& \frac{d y}{d t}=y(-c+2 b x+d y)+\epsilon\left[\lambda_{1} y+\left(\lambda_{2}+\lambda_{3} y\right) \sin \omega t\right] .
\end{aligned}
$$

Our analysis in Sections 2 and 3 in the case where $z_{0}=0$ can be applied to the system (4.1). Hence, from (3.4), if $\lambda_{2} \neq 0$ and

$$
\left|\lambda_{1}\right|<\left|\frac{b+d}{c^{2}} \lambda_{2}+\frac{1}{c} \lambda_{3}\right| \pi \omega \operatorname{cosech}\left(\frac{\pi \omega}{c}\right),
$$

then all three Melnikov functions of the system (4.1) have simple zeros. Hence (4.1) has a transverse heteroclinic cycle for sufficiently small $\epsilon$, so chaotic behaviour exists for sufficiently small $\epsilon$.

THEOREM 4.1. Suppose $\lambda_{2} \neq 0$ and (4.2) holds. Then chaotic behaviour in the sense of Smale exists in the system (4.1) for sufficiently small $\epsilon$.

We note that this result could also be obtained using the standard Melnikov's method for planar systems with a heteroclinic cycle (see [12]). Consider now the case when $b=c=d=0.5, \omega=1, \lambda_{1}=-0.001, \lambda_{2}=1$ and $\lambda_{3}=0$. It is easily verified that these parameter values satisfy the inequality (4.2), so chaotic behaviour exists for sufficiently small $\epsilon$. We present some computer simulations in Figures 3 and 4 where we have chosen $\epsilon=0.05$.

\section{A two-dimensional example}

In this section we provide an application with a model for the self-organisation of macromolecules $[8,9,17]$. We begin by considering a three-dimensional system of Gauss-Lotka-Volterra type which models self-organisation between three molecular species 1,2 and 3 whose densities are $x_{1}, x_{2}$ and $x_{3}$ respectively [17]:

$$
\begin{aligned}
& \frac{d x_{1}}{d t}=x_{1}\left(x_{1}+\alpha x_{2}+\beta x_{3}-M\right), \\
& \frac{d x_{2}}{d t}=x_{2}\left(\beta x_{1}+x_{2}+\alpha x_{3}-M\right), \\
& \frac{d x_{3}}{d t}=x_{3}\left(\alpha x_{1}+\beta x_{2}+x_{3}-M\right),
\end{aligned}
$$




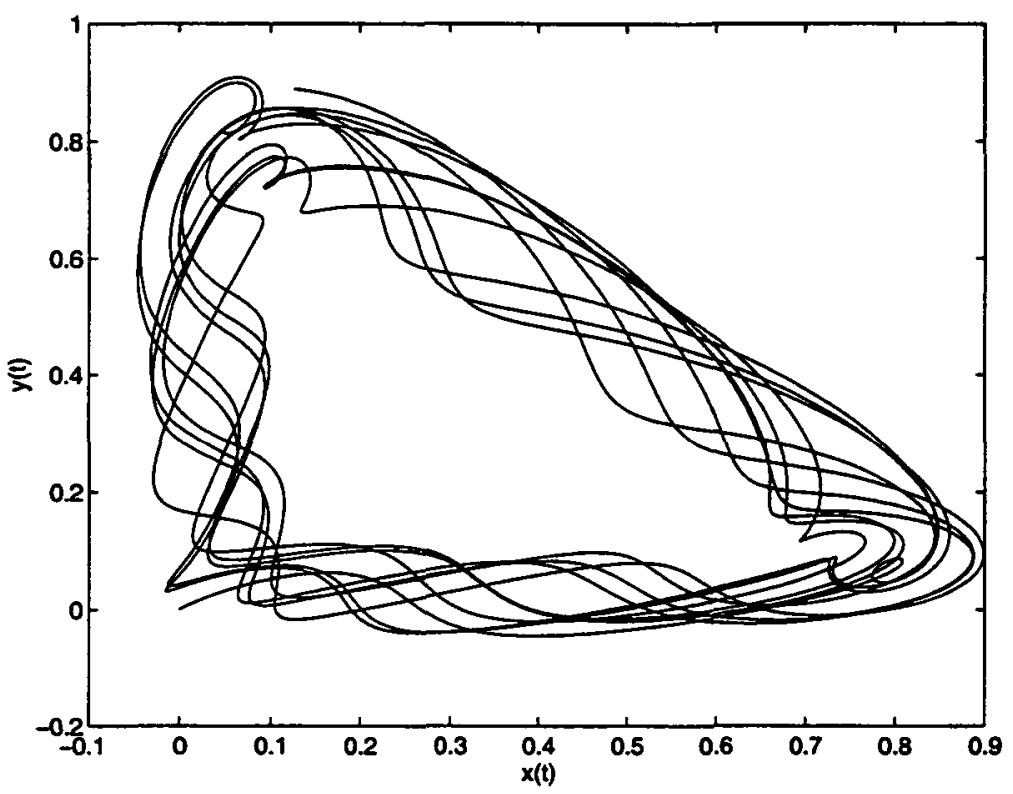

FIGURE 3. An orbit of (4.1) for $b=c=d=0.5, \omega=1, \lambda_{1}=-0.001, \lambda_{2}=1, \lambda_{3}=0$ and $\epsilon=0.05$. The initial value chosen was $(x(0), y(0))=(0,0)$.

where

$$
M=x_{1}\left(x_{1}+\alpha x_{2}+\beta x_{3}\right)+x_{2}\left(\beta x_{1}+x_{2}+\alpha x_{3}\right)+x_{3}\left(\alpha x_{1}+\beta x_{2}+x_{3}\right) .
$$

We consider the case $0<\alpha<1<\beta, \alpha+\beta<2$. If we let $V=x_{1}+x_{2}+x_{3}$, then

$$
\frac{d V}{d t}=M(1-V)
$$

and so orbits in $\mathbb{R}_{+}^{3}=\left\{\left(x_{1}, x_{2}, x_{3}\right) \in \mathbb{R}^{3}: x_{i} \geq 0, i=1,2,3\right\}$ (with the exception of the fixed orbit at the origin) converge as $t \rightarrow \infty$ to the invariant simplex

$$
S_{3}=\left\{\left(x_{1}, x_{2}, x_{3}\right) \in \mathbb{R}_{+}^{3}: x_{1}+x_{2}+x_{3}=1\right\}
$$

We consider the dynamics of the system on $S_{3}$ in the limiting case $\alpha+\beta=2$. Then we can show that the system (5.1) reduces to the two-dimensional system (writing $x \equiv x_{1}$ and $\left.y \equiv x_{2}\right)$

$$
\frac{d x}{d t}=(1-\alpha) x(1-x-2 y)
$$




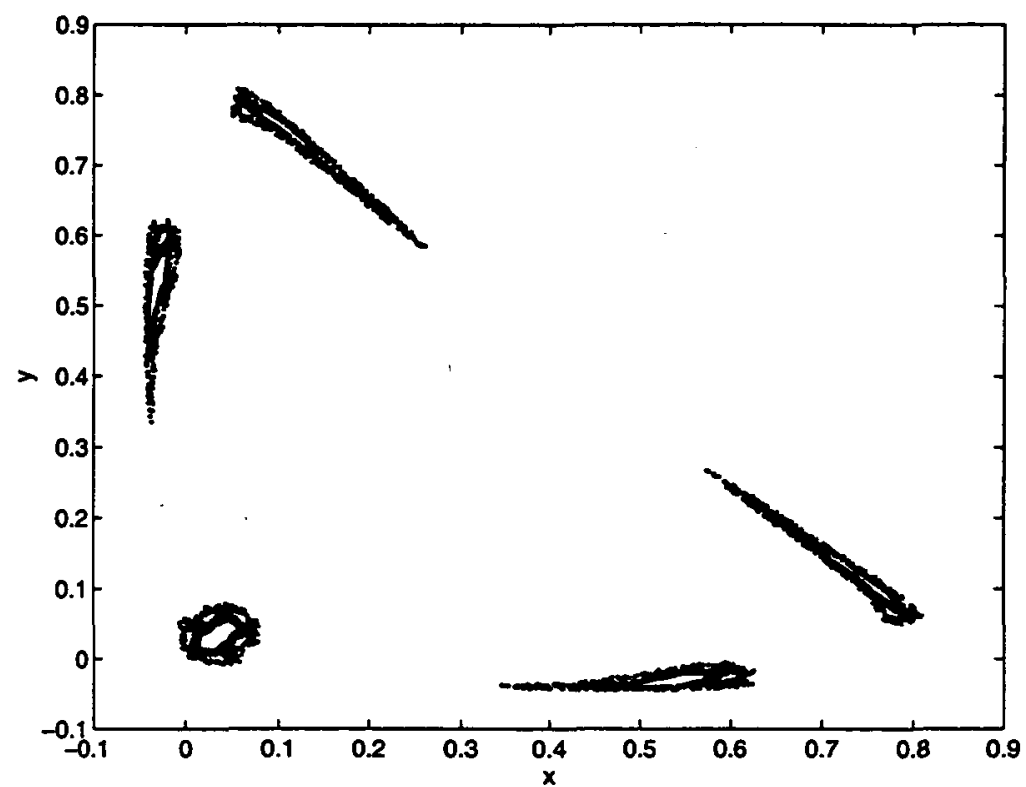

FIGURE 4. Poincare points (minus transients) of (4.1) for $b=c=d=0.5, \omega=1, \lambda_{1}=-0.001, \lambda_{2}=1$, $\lambda_{3}=0$ and $\epsilon=0.05$.

$$
\frac{d y}{d t}=(1-\alpha) y(-1+2 x+y)
$$

This system is identical to the system (4.1) with $\epsilon=0$ and $b=c=d=1-\alpha$. Now consider the perturbed system

$$
\begin{aligned}
& \frac{d x}{d t}=(1-\alpha) x(1-x-2 y)+\epsilon\left[\lambda_{1} x+\left(\lambda_{2}+\lambda_{3} x\right) \sin \omega t\right] \\
& \frac{d y}{d t}=(1-\alpha) y(-1+2 x+y)+\epsilon\left[\lambda_{1} y+\left(\lambda_{2}+\lambda_{3} y\right) \sin \omega t\right]
\end{aligned}
$$

which is identical to the system (4.1) with $b=c=d=1-\alpha$. From (4.2), if $\lambda_{2} \neq 0$ and

$$
\left|\lambda_{1}\right|<\left|2 \lambda_{2}+\lambda_{3}\right| \frac{\pi \omega}{1-\alpha} \operatorname{cosech}\left(\frac{\pi \omega}{1-\alpha}\right),
$$

then chaotic behaviour in the sense of Smale exists in the system (5.2) for sufficiently small $\epsilon$. 


\section{Acknowledgements}

This research was done while the first author was the holder of a Research Science Cadetship from the Defence Science and Technology Organisation (DSTO), Australia. The third author wishes to thank The Flinders University of South Australia for a Visiting Fellowship which enabled the completion of this research.

The authors thank Anna Dostovalova for her helpful suggestions in improving the readability of the paper.

\section{References}

[1] P. F. Byrd and M. D. Friedman, Handbook of elliptic integrals for engineers and scientists (Springer, 1971).

[2] J. R. Christie, K. Gopalsamy and J. Li, "Chaos in sociobiology", Bull. Austral. Math. Soc. 51 (1995) 439-451.

[3] Y.-H. Chu, J.-H. Chou and S. Chang, "Chaos from third-order phase-locked loops with a slowly varying parameter", IEEE Trans. Circuits Syst. 37 (1990) 1104-1115.

[4] J. Coste, J. Peyraud and P. Coullet, "Asymptotic behaviour in the dynamics of competing species", SIAM J. Appl. Math. 36 (1979) 516-543.

[5] M. Farkas, Periodic motions (Springer, New York, 1994).

[6] M. E. Gilpin, "Spiral chaos in a predator-prey model”, Amer. Nat. 113 (1979) 306-308.

[7] J. Guckenheimer and P. Holmes, Nonlinear oscillations, dynamical systems, and bifurcations of vector fields (Springer, New York, 1983).

[8] J. Hofbauer, P. Schuster, K. Sigmund and R. Wolff, “Dynamical systems under constant organisation II: homogeneous growth functions of degree $p=2$ ", SIAM J. Appl. Math. 38 (1980) 282-304.

[9] J. Hofbauer and K. Sigmund, The theory of evolution and dynamical systems (Cambridge University Press, Cambridge, 1988).

[10] M. Inoue and H. Kamifukumoto, "Scenarios leading to chaos in a forced Lotka-Volterra model", Prog. Theor. Phys. 71 (1984) 930-937.

[11] Yu. A. Kuznetsov, S. Muratori and S. Rinaldi, "Bifurcations and chaos in a periodic predator-prey model", Int. J. Bifur. Chaos 2 (1992) 117-128.

[12] J. Li, Chaos and Melnikov's method, (in Chinese) (Chongqing University, Chongqing, 1989).

[13] V. K. Melnikov, "On the stability of the center for time-periodic perturbations", Trans. Moscow Math. Soc. 12 (1963) 1-57.

[14] S. Rinaldi, S. Muratori and Yu. A. Kuznetsov, "Multiple attractors, catastrophes and chaos in seasonally perturbed predator-prey communities", Bull. Math. Biol. 55 (1993) 15-35.

[15] G. C. W. Sabin and D. Summers, "Chaos in a periodically forced predator-prey ecosystem model", Math. Biosci. 113 (1993) 91-113.

[16] W. M. Schaffer, "Order and chaos in ecological systems", Ecology 66 (1985) 93-106.

[17] P. Schuster, K. Sigmund and R. Wolff, "On $\omega$-limits for competition between three species", SIAM J. Appl. Math. 37 (1979) 49-54.

[18] S. W. Shaw and S. Wiggins, "Chaotic dynamics of a whirling pendulum", Physica 31D (1988) $190-211$.

[19] S. W. Shaw and S. Wiggins, "Chaotic motions of a torsional vibration absorber", ASME J. Appl. Mech. 55 (1988) 952-958. 
[20] S. Ushiki, "Central difference scheme and chaos", Physica 4D (1982) 407-424.

[21] S. Ushilki, M. Yamaguti and H. Matano, Discrete population models and chaos, Lecture Notes in Num. Appl. Anal. 2 (1980) 1-25.

[22] S. Wiggins, Global bifurcations and chaos: analytical methods (Springer, New York, 1988).

[23] S. Wiggins, Introduction to applied nonlinear dymamical systems and chaos (Springer, New York, 1990).

[24] S. Wiggins, Chaotic transport in dynamical systems (Springer, New York, 1992).

[25] S. Wiggins and P. Holmes, "Homoclinic orbits in slowly varying oscillators", SLAM J. Math Anal. 18 (1987) 612-629. (See also SIAM J. Math Anal., 19 (1988) 1254-1255, errata.)

[26] S. Wiggins and P. Holmes, "Periodic orbits in slowly varying oscillators", SLAM J. Math. Anal. 18 (1987) 592-611.

[27] S. Wiggins and S. W. Shaw, "Chaos and three-dimensional horseshoes in slowly varying oscillators", ASME J. Appl. Mech. 55 (1988) 959-968. 